\title{
Clinical Spectrum and Resistance Profile of Staphylococcus Infections in a Peri Urban Tertiary Care Hospital
}

\author{
Madhuri $^{1}$, Sweta Jangra ${ }^{2 *}$ (iD) and Manisha Khandait ${ }^{2}$ iD \\ ${ }^{1}$ Allied Health Sciences, SGT University, Gurugram - 122 001, Haryana, India. \\ ${ }^{2}$ Department of Microbiology, SGT University, Gurugram - 122 001, Haryana, India.
}

\begin{abstract}
Staphylococcus may be evaluated to be a serious microorganism to colonizes and contaminate both the healthy and immuno-competent people in the community along with the hospitalized patients with decreased immunity. This bacterium has been commonly present on the skin and in the nasal cavity of the human body. In the particular sites the organisms may give rise to local diseases of the nose, urethra, vagina and gastrointestinal tract, skin however most of them are minor and not associated with any mortality. S. aureus as well as coagulase negative staphylococcus which are gram-positive bacteria that lives on the upper respiratory, mouth and skin structure creation, are the possible element for nosocomial and opportunistic disease in humans as well as in animals. Major complications involved with staphylococcus involved SSI, bullous impetigo, skin \& soft tissues infection, bacteremia, pulmonary infections etc. The situation is further complicated by methicillin resistance in staphylococcus. MRSA/ MRCoNS are considered as the emerging cause of hospital acquired infections. it is very necessary to quantify the load of infections in order to achieve good hospital infection control policies. Furthermore, the morbidity and mortality rate may also be reduced in the same manner.

Keywords: Staphylococcus, peri urban area, S. aureus, Coagulase negative staphylococcus (CoNS), methicillin resistance, infections
\end{abstract}

*Correspondence: swetajangra87@gmail.com; +91 9812681475

(Received: September 10, 2021; accepted: September 28, 2021)

Citation: Madhuri, Jangra S, Khandait M. Clinical Spectrum and Resistance Profile of Staphylococcus Infections in a Peri Urban Tertiary Care Hospital. J Pure Appl Microbiol. 2021;15(4):2163-2169. doi: 10.22207/JPAM.15.4.39

(C) The Author(s) 2021. Open Access. This article is distributed under the terms of the Creative Commons Attribution 4.0 International License which permits unrestricted use, sharing, distribution, and reproduction in any medium, provided you give appropriate credit to the original author(s) and the source, provide a link to the Creative Commons license, and indicate if changes were made. 


\section{INTRODUCTION}

Staphylococcus which are gram- positive bacteria live on the upper respiratory, mouth and skin structure creation, are possible element for nosocomial and opportunistic disease. Firstly isolated in purulent fluid from an abscessed leg by Ogston in the 1880s, further retreat by Rosenbach. ${ }^{1}$

S. aureus and coagulase negative staphylococcus (CONS) are ubiquitous in nature as well as found on the skin and mucous membranes (most often the nasal area) of the healthy individuals as normal human flora. Nasal epithelium is one the most ordinary locations for S. aureus colonization, while transfer on the axilla, perineum and other skin sites appears less often. ${ }^{2}$ Under normal circumstances $S$. aureus and CoNS does not cause any infection in the human subjects; yet, if they are permitted to go into an internal tissue $\&$ bloodstream, these organisms might be a causative agent for variety of potentially critical ailments. $^{3}$

Staphylococcus are most frequently associated with antimicrobial resistance towards different classes of antibiotics which are commonly used to cure such infections. In 1960s Methicillinresistant staphylococcus (MRS) or multidrugresistant staphylococcus strains were initially isolated. ${ }^{4}$

Origin of hospital acquired infections has been found the major responsible cause for quickly progression of various potentially fatal diseases including severe sepsis, necrotizing fasciitis, osteomyelitis, life-threatening pneumonia, endocarditis, and toxinoses such as toxic shock syndrome in the last decade. ${ }^{5}$

Surgical site infection (SSI), folliculitis, staphylococcal food poisoning, bullous impetigo, skin \& soft tissue infections, osteoarticular, bacteremia, Pneumonia, infective endocarditis and catheter associated infections are the major complications associated with staphylococcus. ${ }^{6}$

Healthcare-associated methicillinresistant S. aureus (HA-MRSA) has been significantly related to morbidity \& mortality (longer hospital stays) and may impose significant sustainable responsibility on the healthcare system globally. ${ }^{7}$ In addition the dissemination of methicillin resistance have a great impact on both community and health care settings. ${ }^{8}$
The present study was undertaken to see the disease burden with staphylococcus in a peri urban tertiary care hospital so that the policies can be made for the management of illnesses due to staphylococcus.

\section{MATERIAL AND METHODS \\ Place of study}

The present prospective Study was conducted in the Department of Microbiology, SGT Medical College, Hospital and Research Institute, Gurugram.

\section{Duration of the study}

6 Months.

\section{Data collection}

All the clinical samples received in Microbiology lab in between the period of February, March and April were collected or involved suggestive growth of $S$. aureus/ CONS. Inclusion Criteria

All clinical samples received in the Microbiology laboratory from in and out-patients with suspected Staphylococcus growth were involved in the study.

\section{Specimens}

All the samples received in the microbiology laboratory for routine processing i.e, Urine, blood, pus, CSF, sputum, swabs (skin, conjunctival, wound, throat) were processed for the present study.

\section{Processing of the specimen}

All the samples were processed according to the standard bacteriologically methods i.e, all the samples were inoculated on blood agar followed by incubation at $37^{\circ} \mathrm{C}$. The presumptive colonies were further subjected to biochemical tests in order to identify staphylococci.

\section{Identification of staphylococcus}

Staphylococcus was identified by standard methods i.e, gram staining, culture, catalase test, coagulase test (slide coagulase and tube coagulase). Staphylococcus were further confirmed by novobiocin susceptibility test and culture on Mannitol salt agar (MSA). In addition the staphylococcus isolated from the blood culture of pediatric department were processed with $\mathrm{Vi}$ Tek $2 \mathrm{c}$ identification method as per manufacturers direction. ${ }^{9}$

Antibiotic susceptibility testing

Antibiotic susceptibility testing (AST) 
was done by using disc diffusion method with Mueller-Hinton agar (MHA) as per CLSI 2020 guidelines. Following antibiotics disc containing specific absolute concentration were used: Cefotaxime (30 $\mu \mathrm{g})$, Penicillin (10 units), Ampicillin $(30 \mu \mathrm{g})$, Gentamicin $(10 \mu \mathrm{g})$, Erythromycin $(15 \mu \mathrm{g})$, Tetracycline (30 $\mu \mathrm{g})$, Levofloxacin $(5 \mu \mathrm{g})$,Vancomycin $(10 \mu \mathrm{g})$, Linezolid $(30 \mu \mathrm{g})$, Clindamycin $(2 \mu \mathrm{gm})$,

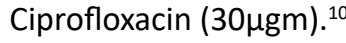

All the samples were discarded according to the latest biomedical norms after completion of the research work.

\section{Statistical analysis}

All the collected data was analyzed by means of Chi-square test which is a suitable analytical method for categorical analysis.

\section{RESULTS}

The present study was designed to be conducted in a peri urban located SGT Hospital, Gurugram Haryana which is a multispecialty tertiary care majorly serving to the rural population. Among the total 270 samples, 142 (52.6\%) were from male patients however 128 (47.4\%) were contributed from female patients.

Samples were received from various departments of the hospital. Highest no of samples were acknowledged from the department of general medicine followed by pediatrics, obstetrics \& gynecology, NICU, general surgery and pulmonary medicine although dermatology and ENT department contributed the same no samples. (Table 1)
Table 1. Samples distribution among all the departments

\begin{tabular}{lcc}
\hline Department & Total & Percentage \\
\hline General surgery & 8 & $2 \%$ \\
General medicine & 79 & $29 \%$ \\
Pediatrics & 68 & $25 \%$ \\
Obstetrics \& gynecology & 51 & $18 \%$ \\
ENT & 14 & $5.1 \%$ \\
Pulmonary medicine & 2 & $0.7 \%$ \\
NICU & 34 & $12 \%$ \\
Dermatology & 14 & $5.1 \%$ \\
\hline
\end{tabular}

Different species of staphylococcus were isolated from all the clinical samples. All the species were categorized on the basis of their ability to produce coagulase enzyme as coagulase positive and coagulase negative staphylococcus. S. aureus was observed as the chief coagulase positive staphylococcus species. In addition S. epidermidis was the commonest coagulase negative staphylococcus species followed by $S$. hominis and S. hemolyticus. (Fig. 1)

Staphylococcus isolation rate was found varies according to the clinical illnesses in the present study. Highest staphylococcus isolation rate was observed from the department of general medicine followed by pediatrics, obstetrics \& gynae. Lowest rate of staphylococcus isolation was observed in the department of pulmonary medicine and ENT. (Table 2)

Highest isolation of staphylococcus was observed from bacterial sepsis (42\%) followed by blood stream infections (38\%), skin and soft tissues infections (10\%). Lowest isolation of

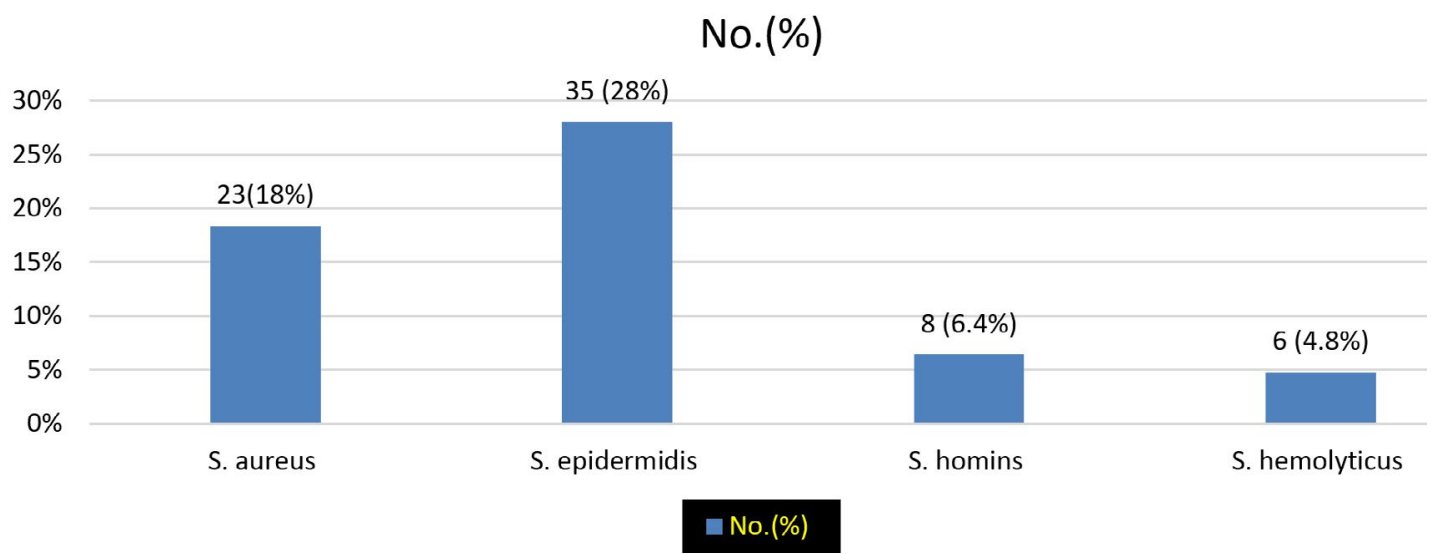

Fig. 1. Species differentiation of all the staphylococcus isolates. 
Table 2. Clinical spectrums of Staphylococcus infections according to departments

\begin{tabular}{lcc}
\hline Department & $\begin{array}{c}\text { Clinical } \\
\text { diagnosis }\end{array}$ & $\begin{array}{c}\text { Staphylococcus } \\
\text { isolation rate No (\%) }\end{array}$ \\
\hline General surgery & SSI & $4(1)$ \\
General medicine & Bacterial sepsis & $31(11)$ \\
Pediatrics & Bacteremia & $28(10)$ \\
Obstetrics \& gynecology & Bacterial sepsis & $22(8)$ \\
ENT & Ocular infections & $7(2)$ \\
Pulmonary medicine & Pulmonary infections & $1(0.3)$ \\
NICU & Blood stream infection & $20(7)$ \\
Dermatology & Skin \& soft tissue infection & $12(4)$ \\
\hline
\end{tabular}

staphylococcus infections was observed from pulmonary diseases (1\%). (Fig. 2)

Highest resistance was observed for penicillin by all the staphylococcus isolates. Coagulase negative staphylococcus showed high resistance towards tetracycline compared to $S$. aureus. Resistance rate towards methicillin were observed high among coagulase negative strains than S. aureus stains. However no significant statistical difference was found in the overall resistance pattern of $S$. aureus and coagulase negative strains. (Table 3)

\section{DISCUSSION}

S. aureus along with other staphylococcus species has been reported as an essential pathogenic organism responsible for several ailments including nosocomial infections. S. aureus has been reported as a specific organism found in pus and blood culture. ${ }^{11}$

The above condition becomes worse with development of multidrug resistance in such pathogenic microorganisms. Many instances of nosocomial infections are associated with opportunistic microorganisms. In case of staphylococcus (S. aureus and CoNS) the situation becomes more difficult with resistance towards methicillin (methicillin-resistant $S$. aureus, MRSA and methicillin resistant CoNS, MRCoNS). ${ }^{12}$

The present study was an attempt to evaluate the antimicrobial susceptibility pattern of staphylococcus from clinical specimens at SGT Medical College, Hospital and Research Institute, Gurugram.

\section{Staphylococcus isolation rate from various} diseases

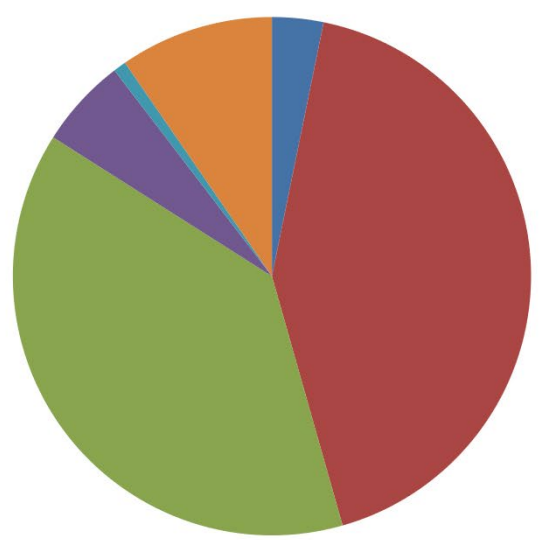

- SSI

- Bacterial sepsis

- Blood stream infections

- Ocular infections

- Pulmonary infections

Skin and soft tissues infections

Fig. 2. Staphylococcus isolation from various diseases 
Table 3. Antibiotic resistance pattern of all the Staphylococcus isolates

\begin{tabular}{lccc}
\hline Antibiotics & $\begin{array}{c}\text { S. aureus } \\
(\mathrm{n}=49)\end{array}$ & $\begin{array}{c}\text { CONS } \\
(\mathrm{n}=76)\end{array}$ & $\begin{array}{c}\text { Statistical } \\
\text { analysis }\end{array}$ \\
\hline Gentamycin & $4(8.1 \%)$ & $7(14.2 \%)$ & $\chi^{2}=0.041, \mathrm{p}>0.001$ \\
Tetracycline & $19(38.7 \%)$ & $23(46 \%)$ & $\begin{array}{c}\chi^{2}=0.968, \mathrm{p}>0.001 \\
\chi^{2}=1.55, \mathrm{p}>0.001\end{array}$ \\
Penicillin & $35(71.4 \%)$ & $46(93.8 \%)$ & $\mathrm{NC}$ \\
Levofloxacin & $1(0 \%)$ & $0(0 \%)$ & $\mathrm{NC}$ \\
Ciprofloxacin & $5(10.2 \%)$ & $3(6.1 \%)$ & $\mathrm{NC}$ \\
Vancomycin & $0(0 \%)$ & $0(0 \%)$ & $\mathrm{NC}$ \\
Erythromycin & $9(18.3 \%)$ & $15(30.6 \%)$ & $\chi^{2}=0.036, \mathrm{p}>0.001$ \\
Clindamycin & $3(6.1 \%)$ & $8(16.3 \%)$ & $\chi^{2}=0.07, \mathrm{p}>0.001$ \\
Cefoxitin & $12(24.4 \%)$ & $17(34.6 \%)$ & $\chi^{*}$
\end{tabular}

${ }^{*} \mathrm{NC}=$ not calculated

Blood, pus and urine sample were collected or received in the microbiology lab as per the standard microbiological guidelines. Most common type of sample was blood and most of the blood culture sample of staphylococcus species were isolated from casualty department followed by general surgery, general medicine, pediatrics, gynecology, casualty, obstetrics, pulmonary medicine. Casualty department showed the highest positivity rate than others since in casualty department there is overburden because of patients, in addition there are very low infection control policies which are followed. However, the lowest positivity rate was observed in the ENT department. On the basis of biochemical tests and automated method of speciation we have isolated different staphylococcus species, among them $S$. epidermidis was the commonest species followed by S. aureus, S. hemolyticus and S.hominis. When we compare our findings with the armed force medical college, they concluded $S$. aureus as the commonest organism isolated from all the specimens which is a discordant result with the present study. ${ }^{13}$

Sex wise distribution of samples or the $M: F$ ratio was not found as significant $(>0.001)$. High number of samples collected/ received form males compared to females which is a concordant finding with another study conducted at Karnataka, India reported high number of samples from male 51 (73\%) compared to females19 (27\%). They showed male have a more infection rate than females. Authors have demonstrated various type of infections as skin and soft tissue infections, respiratory infections, intravascular catheter infections, central nervous system infections. Major complications were reported as sepsis and endocarditis which is again a similar complication observed in our study. On the other hand, this finding does not agree with several other studies who have reported high positivity rate in females as compared to males. In the present study the major isolation rate of staphylococcus was from bacterial sepsis (Fig.2) However further clarifications are still needed in the same direction with large samples as the reason for this difference is unclear. ${ }^{14}$

Methicillin-resistant Staphylococcus aureus (MRSA) is one of the most necessary nosocomial pathogens globally and has been a serious cause of morbidity and mortality in elevated risk wards such as intensive care units. ${ }^{15}$ In some countries, nosocomial $S$. aureus isolates of MRSA account for more than $70 \%$ in Asia. Although, there remains a paucity of details about the MRSA from some of the areas in Asia. In India, there are studies demonstrating that the prevalence of MRSA in the healthcare system is going to increase furthermore MRSA now accounts for a matter of concern nationally. ${ }^{16-18}$

Staphylococcus species showed varied degrees of resistance towards different classes of antibiotics (Table 3 ). Highest resistance was observed for penicillin followed by tetracycline and erythromycin compared to other classes of antibiotics in S. aureus. Furthermore the least resistance was observed for vancomycin and levofloxacin. However CoNS showed markedly 
resistance towards penicillin and tetracycline. Similar resistance pattern was revealed by a study i.e, higher resistance towards penicillin (79\%) followed by oxacillin (58\%). In the present study we observed comparable methicillin resistance in S. aureus and CoNS. As a result, it seems that three antibiotics are showing higher degree of resistance (penicillin, tetracycline and cefoxitin) which is found as a major concern for human health in treating various ailments caused by staphylococcus. ${ }^{19,20}$

MRSA strains which are found resistant to multiple classes of drugs, are called as multidrug resistant staphylococcus which is responsible for high mortality \& morbidity specifically in the ICU's and in immuno-compromised persons. ${ }^{21}$ Methicillin resistance is specifically the change in Penicillin-binding protein to PBP2a. Staphylococcus has been resistance to penicillin/methicillin, which is encoded by the mecA gene. This penicillinbinding protein has been stuck to beta-lactams with lower avidity, that seek difference in the resistance to this group of antimicrobial agents. ${ }^{22}$ Staphylococcus has been reported as a high human pathogen. The virulence of Staphylococcus is due to its ability to produce several virulence factors i.e, $\alpha$ - toxins, penton-valentine leucocidin, enterotoxins, toxic shock syndrome toxins etc. ${ }^{23}$ In this context further studies are needed to elaborate the estimation of infections due to particular virulence factor. In addition there should be a limited use of antibiotics instead irrational use in order to combat the situation of high antimicrobial resistance in India.

\section{CONCLUSION}

Antibiotic resistance is one of the important concerns these days. Some organisms which are considered as commensals but may be associated with serious complications like surgical site infections, bullous impetigo, pneumonia and empyema, infective endocarditis, osteoarticular infections etc.

Among them $S$. aureus is one of the organisms which may be a commensal flora but is associated with many serious complications. The situation is further complicated by antimicrobial resistance in the given organism. Methicillin resistance is the most common resistance encountered in S. aureus. Methicillin resistant strains are very hard to treat as it's a drug of choice for the infections caused by $S$. aureus.

In healthcare system there are many factors for acquisition of hospital associated infections. However among all the pathogens $S$. aureus is one of the most important organisms associated with various hospital acquired infections. MRSA transmission may occur from one patient to another patient in the healthcare system by means of MRSA carriage on the hands of healthcare personnel's.

In order to reduce the chances of such infections its very necessary to take adequate hand hygiene steps along the modified antibiotic stewardship programs to follow the proper hospital infections control policies.

\section{ACKNOWLEDGMENTS}

None.

\section{CONFLICT OF INTEREST}

conflict of interest.

The authors declare that there is no

\section{AUTHORS' CONTRIBUTION}

All authors listed have made a substantial, direct and intellectual contribution to the work, and approved it for publication.

\section{FUNDING}

None.

\section{DATA AVAILABILITY}

The datasets generated during and/or analysed during the current study are available from the corresponding author on reasonable request.

\section{ETHICS STATEMENT}

Not applicable.

\section{REFERENCES}

1. Turner NA, Sharma KB, Maskarinec AA, et al. Methicillin-resistant Staphylococcus aureus: an overview of basic and clinical research. Nat Rev Microbiol. 2019;17(4):203-218. doi: 10.1038/s41579018-0147-4

2. Yang ES, Tan J, Eells S, Rieg G, Tagudar G, Miller LG. Body site colonization in patients with communityassociated methicillin-resistant Staphylococcus 
aureus and other types of $S$. aureus skin infections. Clin Microbiol Infect. 2010;16(5):425-431. doi: 10.1111/j.1469-0691.2009.02836.x

3. Rasheed NA, Hussein NR. Staphylococcus aureus: An Overview of Discovery, Characteristics, Epidemiology, Virulence Factors and Antimicrobial Sensitivity. European Journal of Molecular \& Clinical Medicine. 2021;8(3):1160-1183.

4. Lowy FD. Staphylococcus aureus infections. N Engl J Med. 1998;339(8):520-532. doi: 10.1056/ NEJM199808203390806

5. Monecke S, Coombs G, Shore AC, et al. A field guide to pandemic, epidemic and sporadic clones of methicillin-resistant Staphylococcus aureus. PLoS ONE. 2011;6(4):e17936. doi: 10.1371/journal. pone. 0017936

6. Zoubi SM, Ali Al-Toyyar I, Hussein E, Al Jabali A, Khudairat S. Antimicrobial Susceptibility Pattern of Staphylococcus aureus isolated from clinical specimens in the Northern area of Jordan. Iran J Microbiol. 2015;7(5):265-272. PMID: 26719783

7. Kong EF, Johnson JK, Jabra-Rizk MA. Communityassociated methicillin-resistant Staphylococcus aureus: an enemy amidst us. PLOS Pathogens. 2016;12(10):e1005837. doi: 10.1371/journal. ppat.1005837

8. Chambers HF. The changing epidemiology of Staphylococcus aureus? Emerg Infect Dis. 2001;7(2):178-182. doi: 10.3201/eid0702.010204

9. Mackie T, Collee J, McCartney J. Mackie and McCartney practical medical microbiology. $13^{\text {th }}$ ed., Churchill Livingstone Elsevier. 1989:70-80.

10. CLSI. Performance Standards for Antimicrobial Susceptibility Testing. 30th ed. CLSI supplement M100. Wayne, PA: Clinical and Laboratory Standards Institute; 2020.

11. Thomer L, Schneewind O, Missiakas D. Pathogenesis of Staphylococcus aureus Bloodstream Infections. Annu Rev Pathol. 2016;11:343-364. doi: 10.1146/annurevpathol-012615-044351

12. Singh S, Banerjee G, Agarwal SK, Kumar M, Singh RK. Simple methods for speciation of clinically significant coagulase negative Staphylococci and its antibiotic sensitivity /resistance pattern in ICU of tertiary care center. Biomed Res. 2008 ;19(2):97-101.

13. Singh $\mathrm{CL}$, Cariappa CMP, Das NK. Drug sensitivity pattern of various Staphylococcus species isolated at a tertiary care hospital. Med J Armed Forces
India 2016;72(Suppl-1):62-66. doi: 10.1016/j. mjafi.2016.07.009

14. Mathur P, Kapil A, Das B, Dhawan B. Epidemeology and microbiology of nosocomial bacteremia at an Indian tertiary care hospital. Trop Doct. 2005;35:53-54.

15. Schork NJ, Fallin S, lanchbury JS. Single Nucleotide Polymorphisms and the future of genetic epidemiology. Clinical Genetics. 2000;58(4):250-264. doi: 10.1034/j.1399-0004.2000.580402.x

16. Delorme T, Rose S, Senita J, Callahan C, Nasr P. Epidemiology and susceptibilities of methicillinresistant Staphylococcus aureus in Northeastern Ohio. Am J Clin Pathol. 2009;132(5):668-677. doi: 10.1309/ AJCPQ46ZPQXVHHNC

17. Juayang AC, de Los Reyes GB, de la Rama AJ, Gallega CT. Antibiotic Resistance Profiling of Staphylococcus aureus Isolated from Clinical Specimens in a Tertiary Hospital from 2010 to 2012. Interdiscip Perspect Infect Dis. 2014;2014:898457. doi: 10.1155/2014/898457

18. Klein EY, Sun L, Smith DL, Laxminarayan R. The changing epidemiology of methicillin-resistant Staphylococcus aureus in the United States: a national observational study. Am J Epidemiol. 2013;177(7):666-674. doi: 10.1093/aje/kws273

19. Asangi SY, Mariraj J, Sathyanarayan MS, Nagabhushan, Rashmi. Speciation of clinically significant coagulase negative Staphylococci and their antibiotic resistance patterns in a tertiary care hospital. Int J Bio Med Res. 2011;2(3):735-739.

20. Fiebelkorn KR, Crawdford SA, McElmeel ML, Jorgensen $\mathrm{JH}$. Practical disk diffusion method for detection of inducible clindamycin resistance in Staphylococcus aureus and Coagulase -negative Staphylococci. J Clin Microbiol. 2003;41(10):4740-4744. doi: 10.1128/ JCM.41.10.4740-4744.2003

21. Sheehy SH, Atkins BA, Bejon P, et al. The microbiology of chronic osteomyelitis: prevalence of resistance to common empirical antimicrobial regimens. J Infect. 2010;60(5):338-343. doi: 10.1016/j.jinf.2010.03.006

22. NAIK G, DESHPANDE S.A Study On Surgical Site Infections Caused By Staphylococcus aureus, with A Special Search For Methicillin-Resistant Isolates, Journal of clinical and diagnostic research, 2011; 5(3):502-508.

23. Winn WC, Allen SD, Janda WM, et al. Koneman's color atlas and textbook of diagnostic microbiology: Lippincott Williams \& Wilkins 2018;4(11):65-67. 